\title{
High Accuracy Indoor Imaging Positioning Algorithm Based on Angle Feedback with Visible Light
}

\author{
Hou Wenzuo ${ }^{1, a^{*}}$, Wang Daming ${ }^{2, b}$,Zhang Yankui ${ }^{3, c}$ \\ 1, 2, 3 The PLA Information Engineering University, Zhengzhou City, 450001, China \\ a'wwawanwang@163.com, bwdm123@aliyun.com, '834799435@qq.com
}

Keywords: Imaging Positioning; Angle Feedback; High Accuracy; UKF.

\begin{abstract}
Paper puts forward an imaging positioning algorithm, timely obtaining the acceleration parameters in the inertial frame of triaxial accelerograph of intelligent mobile terminal IMU (Inertial measurement unit) and then taking use of angle feedback principle to indirectly get attitude information of mobile terminal. Through using attitude information as pre-setting parameters which will be involved in imaging positioning UKF (Unscented Kalman Filter) decoding process, restraining the predicted value of observing state to control the range of the optimal solution and avoiding falling into locally optimal solution, the final positioning calculation will be achieved. Theory simulation results show that the proposed algorithm can achieve millimeter-leveled high precision indoor positioning.
\end{abstract}

\section{Introduction}

In recent years, visible light positioning which features its LED popularization, high precision positioning with lighting function, green environmental protection, applying to underground and electromagnetically sensitive areas has become a new research focus in the field of indoor positioning technology. Many scholars both at home and abroad carried out many indoor positioning research based on visible light. According to the different ways of positioning, visible light positioning technology can be classified into non-image positioning and image positioning. By setting up several LED lights emitting data with location information in rooms, optical receivers of the non-image locating can realize positioning according to the intensity of the light signals and the obtained location information. Non-image positioning needs to professional photoelectric detectors. The existing ways are triangulation method, the label positioning method and hybrid network method of sensors. Through terminal imaging components, image positioning gets the labels of LED light source and image information. According to the imaging position relationships between light sources and the focal length of images, image positioning takes use of geometrically optical imaging principles to calculate the terminal position. Existing implementations are single imaging positioning method and multi imaging positioning method.

This paper mainly studies imaging positioning. Multi-imaging positioning method gets different imaging information of LED headlights at the same time through multiple imaging components, and then combines the fixed distance information of imaging components to complete the positioning function. Its positioning precision is high, while it needs a single terminal equipped with multiple imaging components, which need a higher request for correct assembly. Besides, this method needs to develop special terminal equipment. In comparison, the applicability of single image method is much stronger. The intelligent mobile terminals completely meet the requirements in the existing market, but the positioning precision is not ideal. There is still some gap with the requirements of the indoor high precision positioning. Therefore, from the perspective of imaging positioning calculating of visible light, this paper researches how to further improve positioning precision. 


\section{Imaging Positioning Algorithm Based on UKF}

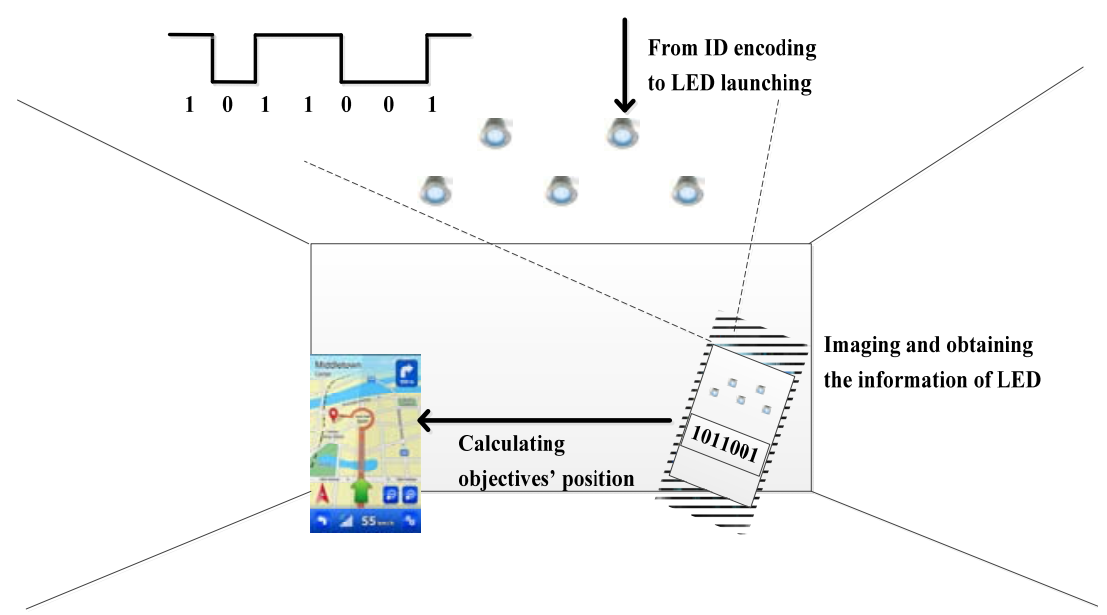

Figure 1 Visable Lighe Imaging Positioning System Model

Through terminal imaging components, this system gets the labels of LED light source and image information in buildings. According to the imaging position relationships between light sources and the focal length of images, image positioning takes use of geometrically optical imaging principles to calculate the terminal position. Its imaging process is in line with the geometrically optical central projection model. Here adopting classical collinearity equation of photogrammetry describes the process:

$$
\left\{\begin{array}{l}
X=-f \frac{a_{1}\left(x-x_{s}\right)+b_{1}\left(y-y_{s}\right)+c_{1}\left(z-z_{1}\right)}{a_{3}\left(x-x_{s}\right)+b_{3}\left(y-y_{s}\right)+c_{3}\left(z-z_{1}\right)} \\
Y=-f \frac{a_{2}\left(x-x_{s}\right)+b_{2}\left(y-y_{s}\right)+c_{2}\left(z-z_{1}\right)}{a_{3}\left(x-x_{s}\right)+b_{3}\left(y-y_{s}\right)+c_{3}\left(z-z_{1}\right)}
\end{array}\right.
$$

Coordinate rotation matrix is as follows:

$$
\left[\begin{array}{lll}
a_{1} & a_{2} & a_{3} \\
b_{1} & b_{2} & b_{3} \\
c_{1} & c_{2} & c_{3}
\end{array}\right]=\left[\begin{array}{lcc}
\cos \varepsilon_{x} \cos \varepsilon_{z}-\sin \varepsilon_{x} \sin \varepsilon_{y} \sin \varepsilon_{z} & -\cos \varepsilon_{x} \sin \varepsilon_{z}-\sin \varepsilon_{x} \sin \varepsilon_{y} \cos \varepsilon_{z} & -\sin \varepsilon_{x} \cos \varepsilon_{y} \\
\cos \varepsilon_{x} \sin \varepsilon_{z} & \cos \varepsilon_{y} \cos \varepsilon_{z} & -\sin \varepsilon_{y} \\
\sin \varepsilon_{x} \cos \varepsilon_{z}+\cos \varepsilon_{x} \sin \varepsilon_{y} \sin \varepsilon_{z} & -\sin \varepsilon_{x} \sin \varepsilon_{z}+\cos \varepsilon_{x} \sin \varepsilon_{y} \cos \varepsilon_{z} & \cos \varepsilon_{x} \cos \varepsilon_{y}
\end{array}\right]
$$

First of all, mobile terminal by receiving the encoding information of LED light source can match world coordinate information of the obtained light source. $\left(x_{j}, y_{j}, z_{j}\right), j=1, \cdots, N, \mathrm{~N}$ stands for the number of lights. After LED imaging of the terminal, the corresponding coordinates of the images of LED light source are $\left(X_{j}, Y_{j}\right), \quad j=1, \cdots, N$. The focal length of imaging lens----f can be obtained through calculation. Through putting the known parameters $\left(X_{j}, Y_{j}, x_{j}, y_{j}, z_{j}, f\right)$ into formula (1) to establish simultaneous system of equations and estimate target's position $\left(x_{s}, y_{s}, z_{s}\right)$ and attitude $\left(\varepsilon_{x}, \varepsilon_{y}, \varepsilon_{z}\right)$ information, collinearity equations (1) have obvious nonlinearity characteristics, it is a good way to use UKF algorithm [10,11,12] to complete the information estimate of target coordinates and attitude coordinates. Set up the nonlinearity system

$$
\left\{\begin{array}{l}
x(k+1)=x(k)+w(k) \\
z(k)=x(k)+v(k)
\end{array}\right.
$$

Assuming that the system state noise $w(k)$ and observation noise $v(k)$ are white gaussian noises and unrelated to each other. The status of system $x(k)$ is $\left(x_{s}, y_{s}, z_{s}, \varepsilon_{x}, \varepsilon_{y}, \varepsilon_{z}\right)$. Observation data $z(k)$ is $\left(X_{j}, Y_{j}\right)_{j \times N}$. 
According to the actual picture A, measured parameters are obtained as shown in Table 1:

Table 1 Picture A Location Date

\begin{tabular}{|c|c|c|}
\hline $\begin{array}{c}\text { World position } \\
\left(x_{j}, y_{j}, z_{j}\right)\end{array}$ & $\begin{array}{c}\text { Pixel coordinates } \\
\text { picture } \mathrm{A}\left(X_{j}, Y_{j}\right)\end{array}$ & $\begin{array}{c}\text { Focal length } \\
f(\mathrm{~mm})\end{array}$ \\
\hline$(0,0,0)$ & $(-3.1760,-2.1917)$ & 9.3 \\
\hline$(418.396,0,0)$ & $(1.4000,-2.2229)$ & 9.3 \\
\hline$(0,240.295,0)$ & $(-3.1539,0.4308)$ & 9.3 \\
\hline$(418.396,240.295,0)$ & $(1.4632,0.3497)$ & 9.3 \\
\hline$(209.198,120.1475,0)$ & $(0.8438,-0.9179)$ & 9.3 \\
\hline
\end{tabular}

Position estimating the pictures A by UKF algorithm, the simulation results are as follows:

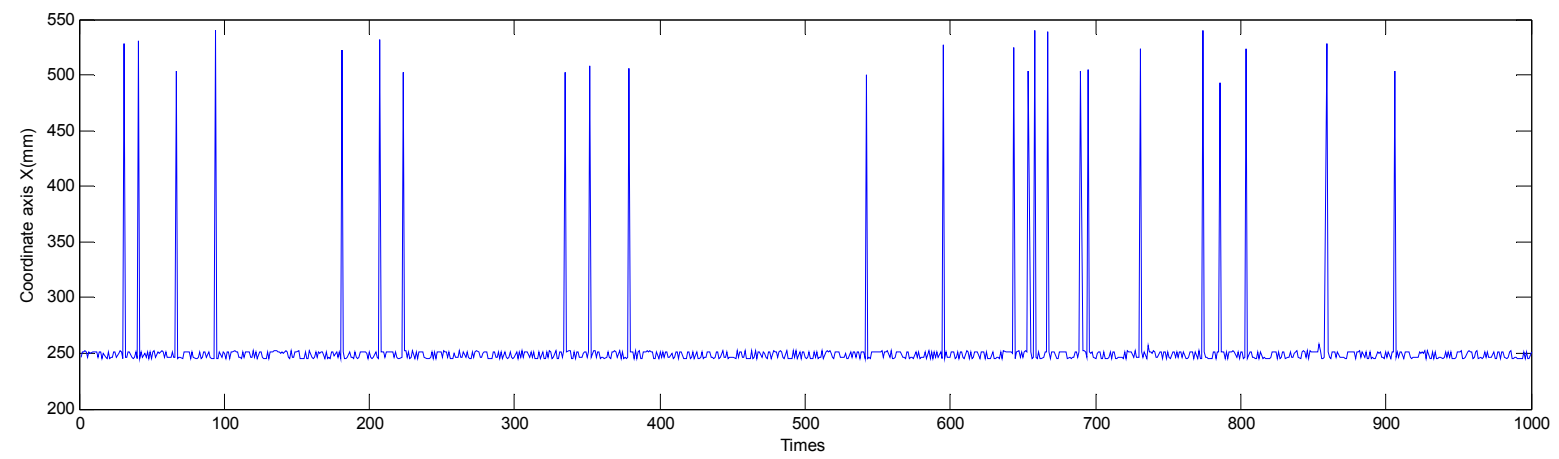

Figure 2 Picture A position estimation results (UKF)

Among 1000 position estimations, it has 24 local optimal solutions. When these 24 abnormal results are eliminated, the errors of positioning results are shown in the following image.

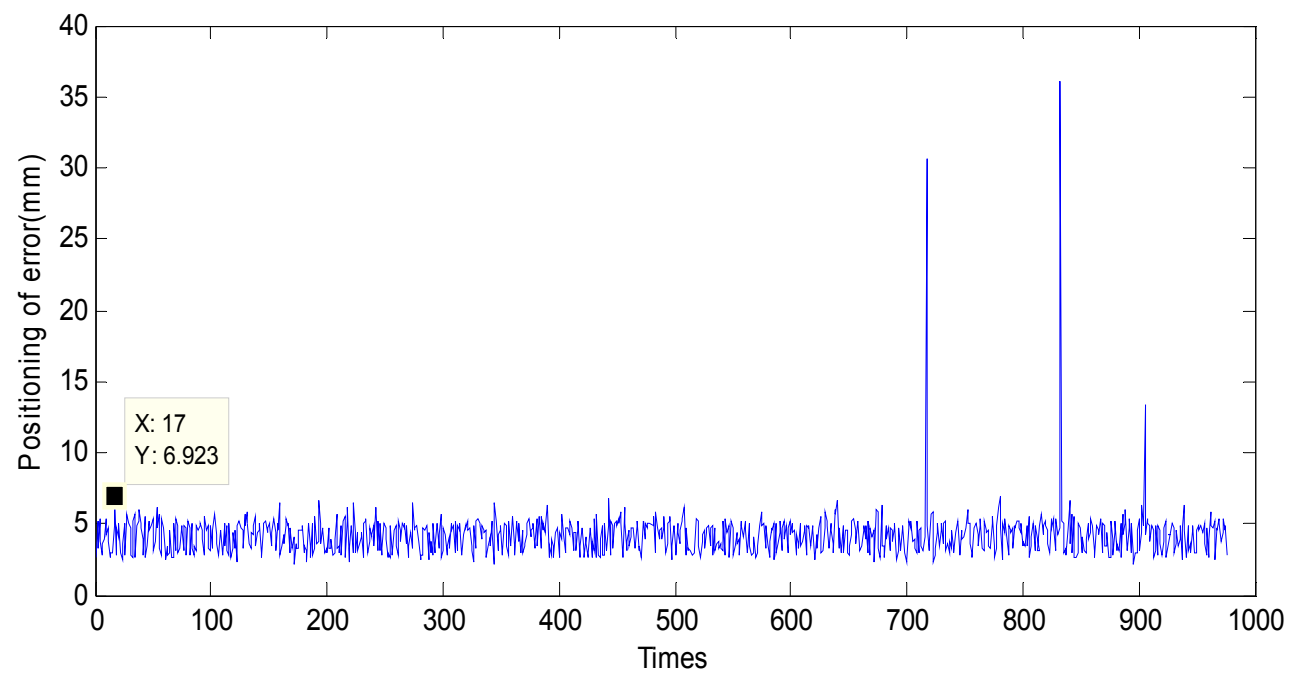

Figure 3 Location estimation error

The simulation results show that among the position information obtained by UFK, 97 percent of the errors will be less than $5 \mathrm{~mm}$ and 3 percent of them will be less than $36 \mathrm{~mm}$.

\section{Angle Feedback}

The result based on the location compute of UKF shows that centimeter-level position accuracy can be achieved in proceeding location compute on the premise of obtaining $\left(X_{j}, Y_{j}, x_{j}, y_{j}, z_{j}, f\right)$ parameters. However, partial location computes may only achieve local optimal solutions. Since the order of magnitude of the positioning accuracy has influence on the application range of the indoor positioning, angle feedback is introduced here in order to improve the compute 
accuracy of the image positioning. All the existing smart mobile terminals are equipped with IMU units whose accelerometers can obtain the acceleration information of terminals $\left(A_{x}, A_{y}, A_{z}\right)$. And then obtains the attitude angle information of the terminals $\left(\varepsilon_{x}, \varepsilon_{y}, \varepsilon_{z}\right)$ by computing. $\left(\varepsilon_{x}, \varepsilon_{y}, \varepsilon_{z}\right)$, as parameter prefix, would be substituted into the solution procedures, which, as the constraint condition, can prevent the compute into local optimal solutions, accelerate convergence speed and further promote positioning accuracy. The terminal attitude information $\left(\varepsilon_{x}, \varepsilon_{y}, \varepsilon_{z}\right)$ can be solved, which includes the pitch angle, roll angle and rotation angle information represented by the terminal in the inertial coordinate system, by the indirect calculation of the tri-axial accelerometer in the IMU. Angle feedback works as follows:

The tri-axial accelerometer can get the real-time acceleration $\left(A_{x}, A_{y}, A_{z}\right)$ of the $\mathrm{X}, \mathrm{Y}$, and $\mathrm{Z}$ axis of the coordinate system where the accelerometer is. Suppose this coordinate system is completely overlap with the World Coordinate System (the inertial coordinate system) where the camera is in.

When the terminal is in the stationary state:

$$
\sqrt{A_{x}^{2}+A_{y}^{2}+A_{z}^{2}}=1 g
$$

According to the definitions of the pitch angle and the roll angle, we can know that:

$$
\begin{aligned}
& \varepsilon_{x}=\arctan \left(\frac{A_{x}}{\sqrt{A_{y}^{2}+A_{z}^{2}}}\right) \\
& \varepsilon_{y}=\arctan \left(\frac{A_{y}}{\sqrt{A_{x}^{2}+A_{z}^{2}}}\right)
\end{aligned}
$$

Electronic compass can directly obtain the rotation Angle of the instrument in information. Electronic compass is unavailable can obtain by gyroscope aided calculation.

\section{The Simulation Results and Analyses}

The attitude information $\left(\varepsilon_{x}, \varepsilon_{y}, \varepsilon_{z}\right)$ of terminal IMU units can be used in UKF calculations as constraints. It is assumed that the attitude information is the optimal one without noise. Under the same simulation conditions in 1.2, the positioning results are shown below:

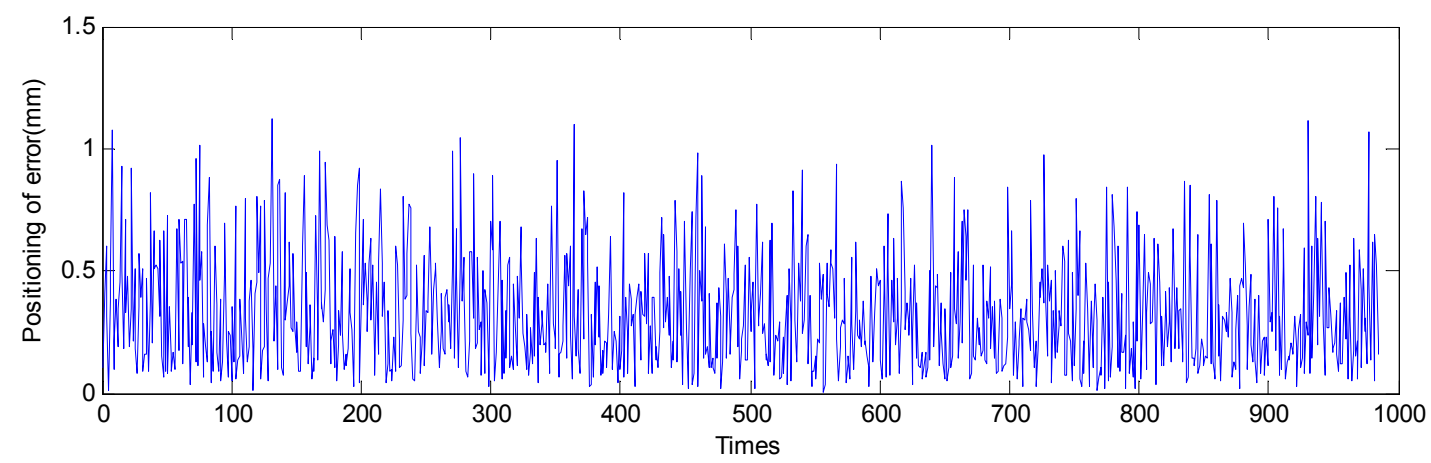

Figure 4 Location estimation error based on angle information

The simulation results show that when angle feedback is adopted, the positioning error will be less than $1.2 \mathrm{~mm}$, thus a high-precision position in millimeter level can be achieved.

\section{Summary}

In this paper, an algorithm, which applies angle feedback to indoor imaging positioning with high precision is proposed. For the UKF imaging positioning calculation of this algorithm, into which the attitude information is involved as pre-setting parameters. Besides, with the control of the optimal solution range via a restriction of predictive values of observational state, a low probability, of which 
UKF might fall directly into the partial optimal solution through a calculation can be achieved maximally. Furthermore, the simulated results illustrated that such algorithm is able to achieve high-precision positioning at millimeter level, which made a further promotion of visible-light imaging positioning that applied to indoor positioning.

\section{References}

[1] Xu Yinfan, Huang Xingxing, Li Rongling, et al. Research on Indoor Positioning Algorithm Based on LED Visible Light Communication [J]. China Light \& Lighting, 2014,(4).

[2] Nah J H Y, Parthiban R, Jaward M H. Visible Light Communications localization using TDOA-based coherent heterodyne detection[C]// Photonics (ICP), 2013 IEEE 4th International Conference on. IEEE, 2013:247-249.

[3] Yamaguchi S, Mai V V, Thang T C, et al. Design and Performance Evaluation of VLC Indoor Positioning System using Optical Orthogonal Codes[C]// Communications and Electronics (ICCE), 2014 IEEE Fifth International Conference on. IEEE, 2014:54-59.

[4] Yong U L, Kavehrad M. Two hybrid positioning system design techniques with lighting LEDs and ad-hoc wireless network[J]. Consumer Electronics IEEE Transactions on, 2012, 58(4):1176-1184.

[5] Masaki Yoshino, Shinichiro Haruyama, Masao Nakagawa. High-accuracy Positioning System using Visible LED Lights and Image Sensor[C]// Radio and Wireless Symposium, 22-24 January, 2008, Orlando. 439-422.

[6] Mohammad Shaifur Rahman, Md. Mejbaul Haque, Ki-Doo Kim. Indoor Positioning by LED Visible Light Communication and Image Sensors[C]// Proceedings of 14th International Conference on Computer and Infonnation Technology, 22-24 December, 2011, Dhaka, Bangladesh. 1-6.

[7] Zhou Qian, Research of the Key Technologis of the Visual Measurement Network System of Single Camera[D], Jilin university,2012.

[8] Han Ping, The Study of Modeling and Simulation Method of Imaging Positioning for Optical Measurement System of Moving Target [D], Harbin Institute of Technology, 2012.

[9] Xiong K, Zhang H Y, Chan C W. Performance evaluation of UKF-based nonlinear filtering[J]. Automatica, 2006, 42(2):261-270.

[10]Wan Li, Liu Yanchun,PI Yiming. Comparing of Target-Tracking Performances of EKF,UKF and PF [J]. Radar Science and Technology, 2007, No.1:13-16.

[11]Pan Quan, Yang Feng, YE Liang, et al. Survey of a kind of nonlinear filiters-UKF[J]. Control and Decision, 2005, No.5:481-489.

[12]Kimberly Tuck. Tilt Sensing Using Linear Accelerometers Freescale Semiconductor Application Note[J].Application Note Rev, 2007, 12:157-164. 